Z Rheumatol 2018 $\cdot 77: 637-638$ https://doi.org/10.1007/s00393-018-0523-5 Online publiziert: 15. August 2018

(c) Springer Medizin Verlag GmbH, ein Teil von Springer Nature 2018

CrossMark

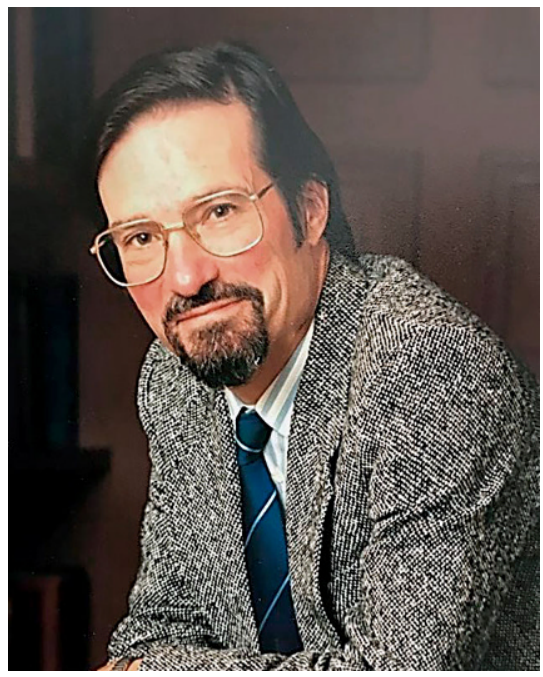

Abb. 1 ^ Prof. Paul Bacon

Paul Bacon, der erste Professor für Rheumatologie in Birmingham, Großbritannien, ist am Freitag, den 12.01.2018 nach kurzer Krankheit friedlich verstorben. Die Deutsche Gesellschaft für Rheumatologie e.V. würdigt seine Leistungen und sein großes Engagement für die Rheumatologie in seiner Heimat, international und auch deren Ausstrahlung auf Deutschland. Dieser Nachruf gibt Teile des „Obituary for Paul Bacon“ (Rheumatology, March 2018, Oxford University Press) wieder.

Paul Bacon war ein wegweisender Rheumatologe, dessen Arbeit maßgebliche Instrumente zur Messung von Outcomes bei Patienten mit systemischen rheumatischen Erkrankungen lieferte. Er war prägend an der Entwicklung der Indizes beteiligt, die eine Quantifizierung von Krankheitsaktivität, -schädigung und -funktionalität ermöglichen. Damit legte er wichtige Grundlagen für die erfolgreiche Bewertung der biolo-

\title{
H.-M. Lorenz
}

Klinik für Hämatologie, Onkologie und Rheumatologie, Universitätsklinikum Heidelberg, Heidelberg, Deutschland

\section{Nachruf zum Tode von Prof. Paul Bacon}

gischen Therapie, die unser Fach so stark gewandelt und der Rheumatologie in den 1990er-Jahren eine neue Richtung gegeben hat. Ohne die von Bacon entwickelten Messungen wären viele der Behandlungen, etwa von Lupus und Vaskulitiden, nicht genehmigt worden. Ein Vermächtnis dieses bedeutenden britischen Rheumatologen ist es sicherlich, bei aller vorhandenen Skepsis von der Notwendigkeit überzeugt $\mathrm{zu}$ haben, Veränderungen zu messen, zu protokollieren und auf diese Wiese eine sichere Grundlage neben der subjektiven klinischen Meinung zu schaffen.

Paul Bacons Ernennung in Birmingham fiel mit der „Geburt“ der Rheumatologie als medizinisches Teilgebiet zusammen. Aufgewachsen in einer Quäkerschule in Reading, ging Bacon nach Cambridge, um Medizin zu studieren und beendete seine klinische Ausbildung am St. Bartholomew's Hospital (Barts) in London. Er selbst soll von sich gesagt haben, dass er das „schwarze Schaf der Familie“ gewesen sei, da er mit der Familientradition von 2 Generationen versicherungsmathematischen Studien brach, um sich der Medizin zu widmen.

Nach seinem Abschluss war er als Assistenzarzt in der Abteilung für Kardiologie in Barts tätig, wo die Herzkatheterisierung in Großbritannien eingeführt wurde. Die Präzision, mit der die Kardiologen die Krankheitsaktivität maßen, so schreiben die Autoren des „Obituary for Paul Bacon“, habe Bacon dazu bewegt, das Gleiche in der Rheumatologie zu wollen, da solche präzisen Werkzeuge bis in die 1980er-Jahre dort nicht existierten. Von 1965 bis 1968 war Bacon wissenschaftlicher Mitarbeiter im neu gegründeten Kennedy Institute in London.
Mit einem Reisestipendium des Arthritis Research Council besuchte er 1971 Carl Pearsons Gruppe an der Universität von Kalifornien in Los Angeles. Auch bei Pearson lernte er das „Messen“; dieses Mal mit Pathologiescores in Tiermodellen mit Arthritis. Bacon kehrte 1972 nach Großbritannien zurück, um Facharzt für Rheumatologie am Royal National Hospital für Rheumatische Erkrankungen in Bath und am Southmead Hospital in Bristol zu werden.

Nachdem er 1989 von einem Sabbatical am National Institute of Health in Bethesda zurückgekehrt, führte Bacon die Abteilung für Rheumatologie in Birmingham in den frühen 1990er-Jahren zu einer Einheit von weltweiter Bedeutung. Mit großem Engagement unterstütze er die Entwicklung des Birmingham Vasculitis Activity Score (BVAS), der 1994 veröffentlich wurde. Gemeinsam mit Loic Guillevin, Wolfgang Gross, Gene Hunder und Cees Kallenberg und mit Caroline Savage und Jo Adu in Birmingham verglich er zum ersten Mal renale und rheumatologische Vaskulitis.

Er war einer der ersten, der rheumatologische Fachkliniken gründete. Mit Forschungsstipendiaten wie David G.I. Scott richtete er eine Klinik für systemische Vaskulitis ein. Dort verglich er die pathologischen Vorgänge bei der Steuerung verschiedener Vaskulitiden. David Scott erinnert sich, wie Paul Bacon ihn in die verschiedenen Krankenhäuser umherschickte, um Patientenkohorten zu sammeln und zu betreuen. Diejenigen, die mit Paul Bacon zusammenarbeiteten, erinnern sich an seine Begeisterung, seine Energie und seine Fähigkeit, Menschen zu überzeugen. 
Paul Bacon interessierte sich nicht nur für klinische Indizes. Großes Interesse zeigt er auch an pathogenen Mechanismen, die rheumatische Erkrankungen begleiten. Er förderte in seiner Einrichtung stark die wissenschaftliche Forschung und stellte sicher, dass alle Mitarbeiter an der Lehre für die Studenten mitwirkten. Er publizierte über 380 wissenschaftliche Paper, die bislang mehr als 20.000-mal zitiert wurden.

Für die Deutsche Gesellschaft für Rheumatologie e. V.

Prof. Dr. Hanns-Martin Lorenz

DGRh-Präsident

\section{Korrespondenzadresse}

Prof. Dr. H.-M. Lorenz

Klinik für Hämatologie, Onkologie und Rheumatologie, Universitätsklinikum Heidelberg

Im Neuenheimer Feld 410, 69120 Heidelberg, Deutschland

Hannes.Lorenz@med.uni-heidelberg.de

\section{Monitor Patientenberatung: Kritik an Kommunikation und medizinischer Beratung}

Kommunikationsdefizite und Schwierigkeiten beim Zugang zur richtigen Therapie inklusive Terminvergabe in Praxen - das sind zwei wesentliche Kritikpunkte, die aus dem "Monitor Patientenberatung 2017" hervorgehen.

Mit dem "Monitor Patientenberatung", der auf der Auswertung der Dokumentation von rund 155.000 Beratungen beruht, hat sich die Unabhängige Patientenberatung Deutschland (UPD) zum Ziel gesetzt, aufzuzeigen, welche Probleme und Herausforderungen Patienten in Deutschland immer wieder zu bewältigen haben. Vor allem zwei große Leitthemen, die im Monitor immer wieder aufscheinen: Kommunikationsdefizite im Umgang mit Patienten und Versicherten und eine vielfach beschriebene Hürde der Ratsuchenden, Ansprüche und Rechte im Alltag wirksam durchsetzen zu können.

\section{Kostengünstige Lösungen?}

Mit Sorge beobachte die Patientenberatung Defizite in der Kommunikation der Kostenträger. So würden für Patienten insbesondere Zwischeninformationen oft wie echte Entscheidungen wirken. Sie fühlten sich von den Kassen dazu gedrängt, die ihnen zustehenden Rechte einer Überprüfung ihrer Ansprüche auf dem Verwaltungsweg nicht auszuschöpfen. Zugleich würden ihnen häufig Lösungen angeboten, die für die Kassen günstiger seien.

\section{Beratungsbedarf bei Krankentagegeld}

Der Auswertung der Beratungsdokumentation zufolge nehmen in der gesundheitsrechtlichen Beratung Fragen zum Krankengeld 2017 eine besonders starke Rolle ein: Die Zahl der Beratungen kletterte von 10.193 Beratungen im Vorjahr auf 14.334 Beratungen. Problematisch seien hier vor allem existenzbedrohende Konsequenzen durch den Verlust des Anspruchs auf Krankengeld aufgrund formaler Lücken in der Arbeitsunfähigkeitsbescheinigung - unabhängig vom objektiven Gesundheitszustand der Versicherten.

Wie im Vorjahr beklagten viele Ratsuchende zudem, dass der Medizinische Dienst der Krankenversicherung (MDK) Begutach- tungen ihres Gesundheitszustandes nach Aktenlage vornehme, heißt es weiter von der UPD. Auch der Verdacht auf Behandlungsfehler (5.851 Beratungen) führe regelmäßig Ratsuchende zur Patientenberatung.

\section{Mangel an qualitativer Info}

In der medizinischen Beratung kritisiert der UPD, dass es offenbar oft an qualitativen Informationen fehle, die den Menschen helfen, die für sie passenden Angebote zu finden. Zudem berichten Ratsuchende von Schwierigkeiten, überhaupt einen Behandlungstermin zu erhalten. In der Arzneimitteltherapie herrschen nach wie vor große Unklarheiten über Zuständigkeiten. All dies trage mit dazu bei, dass Patienten beim Zugang zum Gesundheitswesen scheiterten.

Der Patientenbeauftragte der Bundesregierung bezeichnete die geschilderten Problemlagen als Anstoß für konkrete Verbesserungen. Er verwies dabei auf die schon jetzt bestehenden Aufklärungs- und Informationspflichten und auch auf die Anstrengungen der Politik, etwa mit der Ausweitung der Sprechstunden in den Arztpraxen und einer Verbesserung der Arbeit der Terminservicestellen schon beim Zugang zum Gesundheitssystem anzusetzen.

Quelle: Unabhängige Patientenberatung Deutschland www.patientenberatung.de 